JPP 2008, 60: 943-950 (C) 2008 The Authors

Received December 21, 2007

Accepted March 7, 2008

DOI 10.1211/jpp.60.8.0001

ISSN 0022-3573
Center for Radiological Research, College of Physicians and Surgeons, Columbia University, New York, NY 10032, USA

Tom K. Hei, Hongning Zhou, Vladimir N. Ivanov, Mei Hong, Howard B. Lieberman, David J. Brenner, Sally A. Amundson,

Charles R. Geard

Department of Environmental Health Sciences, Mailman School of Public Health, Columbia University, New York, NY 10032, USA

Tom K. Hei, Howard B. Lieberman, David J. Brenner

Correspondence: Professor Tom K. Hei, Center for Radiological Research, Columbia University, vC11-204, 630

West 168th Street, New York, NY 10032, USA. Email: tkh1@columbia.edu

Acknowledgements: Work supported by funding from the National Institutes of Health (grants CA 49062, CA 130536, ES 11804), NIH Resource Center (grant RR 11623), Environmental Center (grant ES 09089) and the low dose program of the Department of Energy (grant DE-FG02-03ER63441).

\section{Mechanism of radiation-induced bystander effects: a unifying model}

\author{
Tom K. Hei, Hongning Zhou, Vladimir N. Ivanov, Mei Hong, \\ Howard B. Lieberman, David J. Brenner, Sally A. Amundson \\ and Charles R. Geard
}

\begin{abstract}
The radiation-induced bystander effect represents a paradigm shift in our understanding of the radiobiological effects of ionizing radiation, in that extranuclear and extracellular events may also contribute to the final biological consequences of exposure to low doses of radiation. Although radiation-induced bystander effects have been well documented in a variety of biological systems, the mechanism is not known. It is likely that multiple pathways are involved in the bystander phenomenon, and different cell types respond differently to bystander signalling. Using cDNA microarrays, a number of cellular signalling genes, including cyclooxygenase-2 (COX-2), have been shown to be causally linked to the bystander phenomenon. The observation that inhibition of the phosphorylation of extracellular signal-related kinase (ERK) suppressed the bystander response further confirmed the important role of the mitogen-activated protein kinase (MAPK) signalling cascade in the bystander process. Furthermore, cells deficient in mitochondrial DNA showed a significantly reduced response to bystander signalling, suggesting a functional role of mitochondria in the signalling process. Inhibitors of nitric oxide (NO) synthase (NOS) and mitochondrial calcium uptake provided evidence that NO and calcium signalling are part of the signalling cascade. The bystander observations imply that the relevant target for various radiobiological endpoints is larger than an individual cell. A better understanding of the cellular and molecular mechanisms of the bystander phenomenon, together with evidence of their occurrence in-vivo, will allow us to formulate a more accurate model for assessing the health effects of low doses of ionizing radiation.
\end{abstract}

\section{Introduction}

Radiation is a double-edged sword: on the one hand it is an effective therapeutic modality for the treatment of many types of human cancers; on the other hand, it is a well-known human carcinogen. The lifetime cancer mortality risk from radiation, based on epidemiological data from survivors of the Japanese atomic bombs, is estimated to be 0.05 per sievert (ICRP 2005). However, direct characterization of risk at lower doses is at or beyond the limits of epidemiology. Cancer risks from exposure to ionizing radiation clearly increase at doses above 10 cGy, and no obvious threshold dose is detectable (Preston 2005).

At doses below $10 \mathrm{cGy}$, the radiobiological effects are rather complex and are subject to modulations by various competing forces, including bystander effects.

The radiation-induced bystander effect is defined as the induction of biological effects in cells that are not directly traversed by a charged particle, but are in close proximity to cells that are. Interest in this effect was sparked by earlier reports demonstrating that, following a low dose of $\alpha$-particles, a larger proportion of cells showed biological damage than was estimated to have been hit by an $\alpha$-particle based on the microdosimetric principle (Nagasawa \& Little 1992). Specifically, 30\% of the cells showed an increase in sister chromatid exchanges, even though fewer than $1 \%$ were calculated to have undergone a nuclear traversal. It is reasonable to assume that the non-hit cells in the vicinity of a hit cell contributed to the induction of biological damage, or a bystander effect. However, the number of cells hit was estimated by a calculation, based on the fluence of $\alpha$-particles and the crosssectional area of the cell nucleus. The conclusion was thus based on a statistical argument, 
since it was not possible to know which cells were hit on an individual basis and which were not.

The development of single-particle microbeams, which allow a single cell and/or a subcellular compartment to be irradiated selectively with one or multiple particles, has greatly facilitated our understanding of the bystander phenomenon. To demonstrate the induction of a radiation-induced bystander effect unequivocally, studies were conducted using a microbeam where a defined proportion of cells in a confluent monolayer were irradiated individually with a lethal dose of $20 \alpha$-particles (Zhou etal 2000). Since dead cells could not produce mutants, the progeny of the irradiated population, in the absence of any interaction between the hit and non-hit cells, should result in a mutant fraction that was comparable to the non-hit cells (i.e. background levels). In actuality, when the experiments were completed it was clear that the non-clonogenically viable cells had influenced the mutant fraction of the non-hit cells, since the incidence among the progeny was 3-4 times higher than expected (Figure 1). This study provided the first clear-cut indication of a radiation-induced bystander phenomenon.

The bystander effect clearly illustrates that direct damage to the DNA of a neighbouring cell is not necessary for radiationinduced heritable effects. In retrospect, evidence for this nontargeted effect has been available for more than six decades. As early as the 1940s, there were reports that the inactivation of biological entities could be brought about equally by ionizations produced within the entity or by ionization of the surrounding medium (Dale 1940; Lea et al 1944). By 1947, Kotval and Gray had shown that $\alpha$-particles that pass close to the chromatid thread, as well as those that pass through it, have a significant probability of producing chromatid and isochromatid breaks or chromatid exchanges.

\section{Radiation-induced bystander studies using microbeams}

With the availability of charged-particle microbeams, studies have been conducted with both confluent as well as sparsely populated human and other mammalian cells using a variety of biological endpoints. Both $\alpha$-particles and protons had been used where a bystander effect was observed among non-hit cells in which either a single or a defined proportion of cells was targeted. Furthermore, increasing the number of particle traversals per cell (Zhou et al 2001; Shao et al 2003) or the total dose delivered to the irradiated fraction (Hu et al 2006) did not increase the intensity of the bystander response. Thus, there is no evidence of a dose response in the bystander effects. Furthermore, addition of the calcium blocker calcicludine (Shao et al 2006) or NS-398, an inhibitor of cyclooxygenase (COX)-2, significantly reduce the bystander response in human lung fibroblasts (Zhou et al 2005).

\section{Medium-transfer-mediated bystander effects}

Earlier studies have shown that transfer of medium from an irradiated culture to non-irradiated cells can induce increased biological effects in the latter. Mothersill and Seymour (1997) first demonstrated a highly significant reduction in cloning efficiency in both non-irradiated normal cells as well as malignant epithelial cell lines that had received medium from ${ }^{60} \mathrm{Co}$-gamma-ray irradiated cultures. These results suggested that irradiated cells secreted a cytotoxic factor into the culture medium that was capable of killing non-irradiated cells. Furthermore, transferring medium from cultures irradiated with low linear energy transfer to un-irradiated cells increases the levels of various bystander effects, such as cell killing (Lyng etal 2000; Nagar etal 2003), neoplastic transformation (Lewis etal 2001) and genomic instability (Mothersill \& Seymour 1997). As shown in Figure 2, several adaptations of the medium-transfer approach have been used over the years, utilizing a variety of biological endpoints.

To ascertain whether irradiated medium, with or without accompanying cell cultures, can induce bystander genotoxic endpoints in a human hamster hybrid $\left(\mathrm{A}_{\mathrm{L}}\right)$ cell line, customdesigned double mylar dishes were used. One side (with or without cells, Figure 2) was irradiated with $\alpha$-particles using a

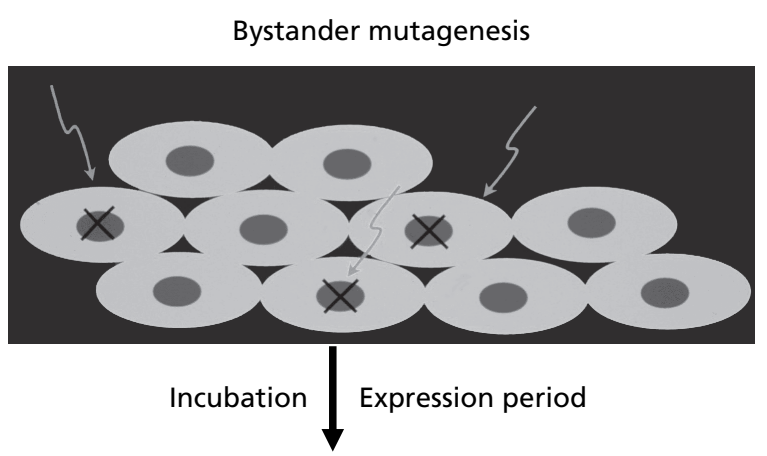

Absence of bystander yields background mutation frequency

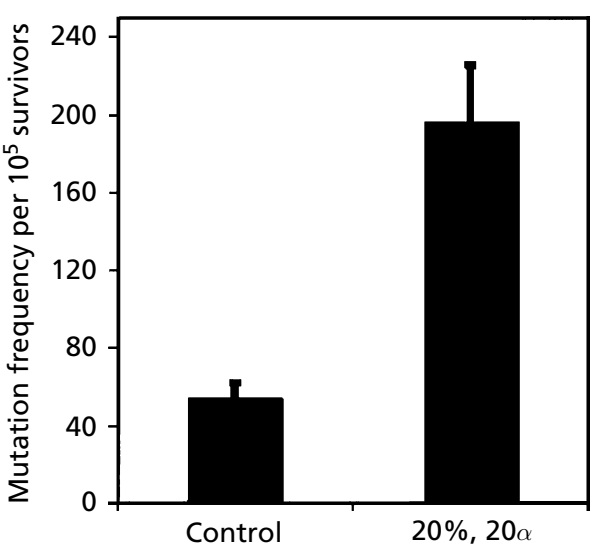

Figure 1 The unequivocal demonstration of the presence of a bystander mutagenic effect in the human hamster hybrid $\left(\mathrm{A}_{\mathrm{L}}\right)$ cells using a charged particle microbeam in which $20 \%$ of the cells were lethally irradiated with $20 \alpha$-particles each through the nucleus. Assuming that there was no interaction between the irradiated-but-dead cells and the non-irradiated cells, the resultant mutant fraction from the irradiated population should be similar to the spontaneous or background level. In reality, as a result of the cross-talk between the hit and non-hit cells, the resultant mutant fraction in the irradiated population was three-fold higher than the background yield (refer to Zhou et al 2000 for detail). 


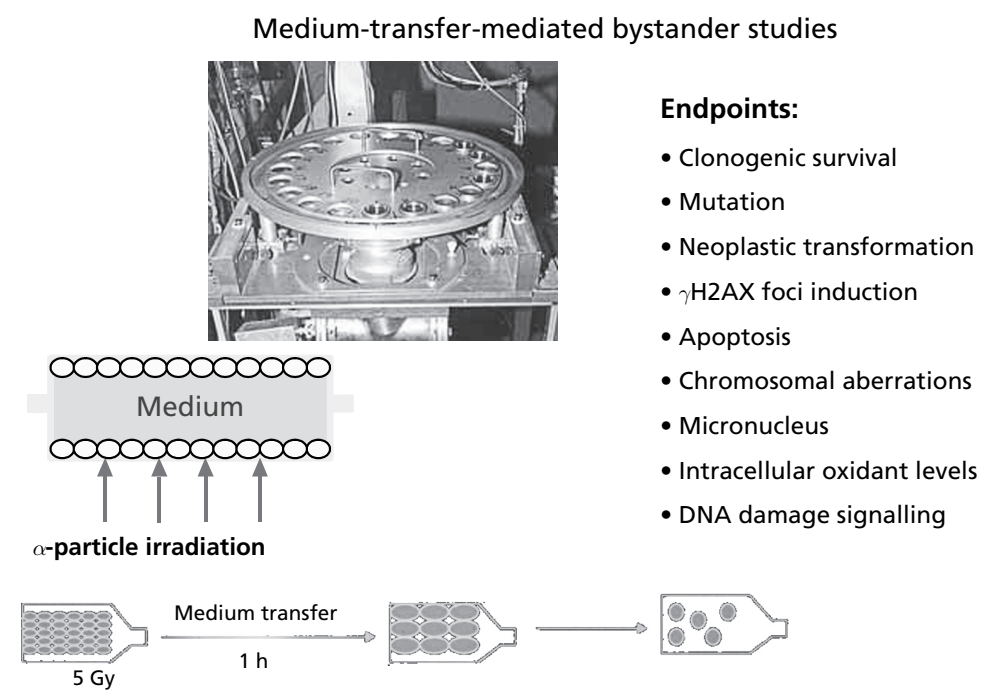

Figure 2 The various approaches used to demonstrate bystander endpoints using medium-transfer experiments. The top panel illustrates track segment irradiation at the Radiological Research Accelerator Facilities of Columbia University (www.raraf.org). Cells plated on stainless steel rings with a $6 \mu \mathrm{m}$ mylar bottom were exposed to a parallel broad-area beam of monoenergetic helium-3 ions. The ions deposited a fraction of their energy in the target cells and the average linear energy transfer over the segment of the track that penetrated the target was around $90 \mathrm{keV}_{\mu} \mathrm{m}^{-1}$. The middle panel shows a 'double mylar' design (Geard et al 2002; Zhou et al 2002) in which irradiated cells plated on the lower mylar bottom secreted soluble mediators into the medium, which modulate the biological behaviour of the non-irradiated cells plated on the upper panel, $\alpha$-particles having little penetrance. The bottom panel shows the classic medium-transfer experiment as described by Mothersill \& Seymour (1997).

$\gamma \mathrm{H} 2 \mathrm{AX}$, phosphorylated histone H2AX (a protein often associated with DNA double-strand breaks).

broad beam from the track segment mode of a $4 \mathrm{MeV}$ Van de Graaff accelerator (Zhou et al 2002). Since $\alpha$-particles can traverse only a very limited distance, cells plated on the other side of a medium-filled mylar dish would not be irradiated by the particles. Non-irradiated target cells attached to the top mylar layer were found to have a much higher number of chromatid-type aberrations when there was a bottom layer of cells in the medium-filled chambers than when just medium alone was present (Suzuki et al 2004). In fact, very few chromatin fragments were induced in the non-hit bystander cells in the top layer when only medium were irradiated. This increase in bystander chromatid breaks showed a time-dependent factor, since the incidence increased with increasing incubation period (Suzuki et al 2004). Furthermore, when transferring the medium from these cell-irradiated dishes to fresh $A_{L}$ cultures, chromatid-type aberrations were produced in the unirradiated cells. Using the same experimental set up, Zhou et al found no induction of $\mathrm{CD} 59^{-}$mutations but an increase in cytotoxicity under similar experimental conditions (Zhou et al 2002). These results suggest that certain, as-yet-unidentified, excreted factor(s) from the irradiated cells on the bottom mylar layer induce some non-repairable chromosomal changes, resulting in an increased incidence of chromatid breaks. However, there was no increase in mutagenesis, presumably as a consequence of increased cell death among the putatively mutated bystander cells.

\section{Mechanism of the bystander response}

Previous studies using the Columbia microbeam showed that irradiating $5 \%$ of a confluent monolayer of cells with a single $\alpha$-particle resulted in a bystander mutagenic yield that was $58 \%$ of the yield when all the cells were irradiated. It is of interest to note that there was no difference in the yield of mutants when the fraction of irradiated cells increased from $10 \%$ to $100 \%$. This could be because the fraction of nonirradiated cells in the population that were in direct contact with, and affected by, an irradiated cell had reached a plateau at $10 \%$ and suggests that cell density is important in bystander mutagenesis.

Since cell-density dependence of the bystander effect implies cell-to-cell contact in the process, the relationship between gap junctional activity and $\alpha$-particle-induced bystander mutagenicity was investigated in two ways: (1) the use of drugs such as lindane and octanol to inhibit gap-junction-mediated intercellular communication; (2) using genetically engineered cells that lack gap junctions. In the first approach, several laboratories, including our own, have shown that treatment of cells with non-toxic doses of either lindane or octanol suppressed the bystander response for a variety of endpoints (Azzam et al 1998, 2000; Zhou et al 2000; Lyng et al 2000; Shao etal 2003; $\mathrm{Hu}$ etal 2005). Although these results indicate a role of gap junctions in the bystander response, octanol and lindane are non-specific inhibitors of gap junctions, and can have wide-ranging effects on other cellular structures and functions, including membrane fluidity (Dowling-Warriner \& Trosko 2000). Therefore, to investigate more specifically the role of gap-junction-mediated cell-to-cell communication in $\alpha$-particle-induced bystander mutagenicity, it is necessary to use cells in which gap junctional activity is suppressed by a dominant negative connexin construct. 


\section{Dominant negative connexin $\mathbf{4 3}$ cells show no bystander genotoxic responses}

Connexin 43 is the principal protein component of gap junctions and there is good evidence that connexin itself (assembled in a lipid bilayer) is sufficient and necessary for the generation of gap junction channels (Bruzzone \& Meda 1998; Trosko 1998). Using the standard scrape-loading test as a measure of gap junctional activity (El-Fouly et al 1987), it was found that migration of Lucifer yellow was completely blocked in $A_{L}$ cells carrying the dominant negative connexin 43 construct (Zhou et al 2001). In contrast, the dye was found to migrate many cell layers in distance among wild-type $A_{L}$ cells, as well as in cells carrying a connexin 43 overexpressing construct. In contrast, there was little, if any, bystander effect among cells carrying the dominant negative vector. These data clearly show that the connexin 43 construct is working well in the transfected cells and that gap junction intercellular communication is critical in mediating the bystander mutagenic process.

\section{Cyclooxygenase- 2 as a central component of the bystander signalling scheme}

A novel mylar design consisting of two concentric stainless rings fitted with mylar bottoms of different thickness has been used to study signalling cascades in bystander cells. The outer ring is fitted with a thin $(6 \mu \mathrm{m})$ mylar bottom and the inner ring has thicker $(38 \mu \mathrm{m})$ strips of mylar, thereby creating a bystander population of cells located next to directly hit cells in a confluent culture. Experiments have been conducted with normal human lung fibroblasts (NHLF) to identify genes that are expressed differently in directly irradiated and bystander cells (Zhou et al 2005). Among the 96 genes represented on the platform, the abundance of one message, $\mathrm{COX}-2$, was found to be consistently higher by more than three-fold, while the RNA level of insulin growth factor binding protein-3 (IGFBP3) was found to be consistently lower by more than seven-fold in several analyses of multiple bystander samples (Figure 3). Semi-quantitative RT-PCR was used to confirm the expression levels of these two genes, by comparing expression levels with that of the glyceraldehyde 3-phosphate dehydrogenase $(G A P D H)$ gene as an internal control. Levels of the COX-2 protein in the non-irradiated bystander cells were further confirmed by Western blotting. Addition of the COX-2 inhibitor NS-398 $(50 \mu \mathrm{M})$ suppressed COX-2 activity in NHLF cells and bystander mutagenesis at the hypoxanthine guanine phosphoribosyltransferase (HPRT) locus (Zhou et al 2005). These results indicated that expression of COX-2 is associated with the bystander effect.

\section{Elucidation of the nature of the signalling molecule}
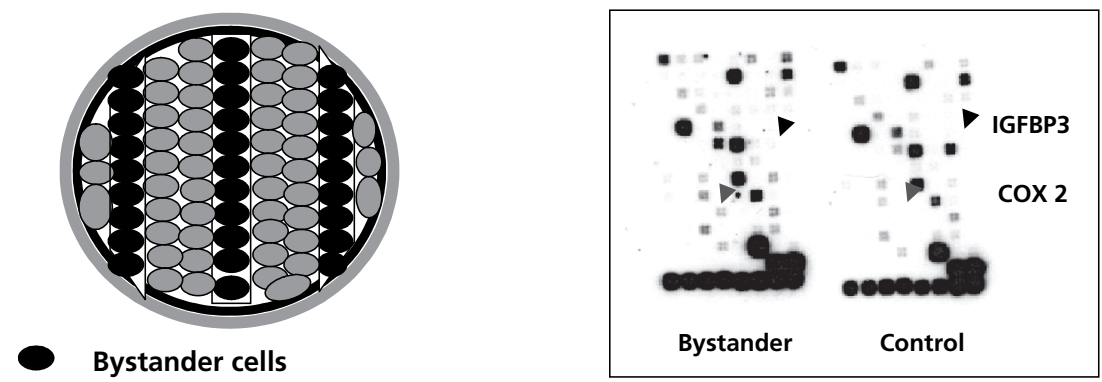

Effect of COX-2 inhibitor on bystander mutagenesis

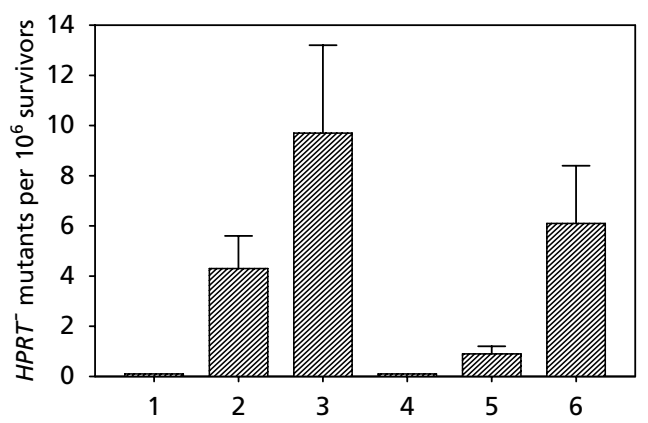

1. Control

2. Bystander

3. $0.5 \mathrm{~Gy} \alpha$-particle

4. NS $398(50 \mu \mathrm{M})$

5. NS398 + bystander

6. NS398 $+\alpha$-particle

Figure 3 The use of a novel 'double mylar' design in which both directly irradiated and bystander cells were cultured on the same vessel. A pathway-specific array was used to identify differentially expressed genes among either non-irradiated or bystander fractions of the double mylar cultures. Two genes were found to be differentially expressed in the bystander cells: a three-fold increase in cyclooxygenase (COX)-2 and a seven-fold decrease in insulin growth factor binding protein-3 (IGFBP-3). To illustrate the functional correlation of COX-2 over-expression with bystander mutagenesis, cultures were concurrently exposed to NS398, a COX-2 inhibitor, which suppressed the bystander mutagenic response (refer to Zhou et al 2005 for detail).

HPRT, hypoxanthine guanine phosphoribosyltransferase. 
A decrease in IGFBP3 level increases the binding of insulin growth factor to cell surface receptors, which activate the downstream signalling events, including, among others, mitogen-activated protein kinase (MAPK). Activation of extracellular signal-related kinase (ERK) by phosphorylation is a critical upstream event preceding $C O X-2$ expression. Previous studies have shown a strong upregulation of phospho-ERK levels in bystander NHLF cells $4 \mathrm{~h}$ after treatment, and that the ratio of phosphorylated ERK relative to native ERK increased from 2 to 13 among bystander cells (Zhou etal 2005). The observations that addition of PD98059 $(50 \mu \mathrm{M})$, a specific inhibitor of MEK-ERK (MEK is the immediate upstream activator of ERK), to the culture medium suppressed bystander effects provided further evidence of the role of ERK in the signalling scheme. Using the mediumtransfer approach, similar findings on the role of ERK signalling pathways have also been documented with immortalized human keratinocytes (Lyng et al 2006).

\section{Role of nuclear factor (NF)- $\kappa$ B in the bystander response}

Since $\mathrm{NF}-\kappa \mathrm{B}$ is an important transcription factor for many signalling genes, including $C O X-2$, it is likely that $\mathrm{NF}-\kappa \mathrm{B}$ participates in the bystander response. There is clear evidence that $\alpha$-particle irradiation upregulates NF- $\kappa \mathrm{B}$ binding activity in both directly irradiated and bystander cells, while Bay 11-7082, a pharmacological inhibitor of $\mathrm{I} \kappa \mathrm{B}$ kinase (IKK)/ $\mathrm{NF}-\kappa \mathrm{B}$, efficiently suppresses this up-regulation and also reduces levels below the basal amount (Zhou et al 2008). This inhibitor of NF- $\kappa \mathrm{B}$ activity also efficiently down-regulates expression of $C O X-2$ and inducible nitric oxide synthase $(i N O S)$ in both directly irradiated and bystander fibroblasts. Earlier studies using confluent human skin fibroblasts exposed to low fluences of $\alpha$-particles show a rapid up-regulation of NF- $\kappa \mathrm{B}$, c-Jun N-terminal kinase (JNK) and ERK in the exposed population (Azzam et al 2002) and suggests activation of these stress-inducible signalling pathways in bystander cells. Furthermore, addition of the antioxidant superoxide dismutase (SOD) was found to suppress the induction. Since induction of $\mathrm{NF}-\kappa \mathrm{B}$ binding activity can be found in both directly irradiated and bystander cells, its role in the bystander response in this study is equivocal.

\section{Effects of cytokines on the bystander response}

There is recent evidence that exogenous tumour necrosis factor (TNF) $\alpha$ in concert with interleukin (IL) $-1 \beta$ directly controls $C O X-2$ expression in NHLF (Zhou et al 2008). Both $\mathrm{TNF} \alpha$ and IL- $1 \beta$ were found to be induced following $\alpha$-irradiation of NHLF. Introduction of inhibitory monoclonal antibodies $(\mathrm{mAb})$ against $\mathrm{TNF} \alpha$ into the cell media substantially decreased levels of NF- $\kappa \mathrm{B}$ and JNK, which was accompanied by a pronounced decrease in $C O X-2$ expression in both irradiated and, especially, bystander NHLF (Zhou et al 2008). Furthermore, there is evidence that addition of anti-TNF mAb has a suppressive role on ERK activity among bystander cells (Zhou et al 2008). These studies provide a clear link between the binding of cell surface receptors for the various cytokines and the downstream activation of NF- $\kappa \mathrm{B}$ and MAPKs.

\section{Role of reactive radical species in the bystander response}

Reactive oxygen species, including hydroxyl radicals and superoxide anions, have been implicated in various mediummediated bystander responses using a variety of endpoints (Azzam etal 1998, 2002; Yang et al 2005; Hu etal 2006; Lyng et al 2006). Since almost all reactive oxygen species have relatively short half-lives, in order for them to be relevant it is likely that they are generated either very close to the target sites or are produced through a continuous cascade of events. There is evidence that the radical-generating scheme of NADPH oxidase is involved in the bystander response (Narayanan et al 1997; Azzam et al 2002). Furthermore, these short-lived, highly reactive radical species have been postulated to be important in the secondary generation of long-lived organic radicals that cause mutations and transformation in human cells (Koyama et al 1998).

The role of reactive nitrogen species, particularly NO, in the bystander response has been investigated extensively using a variety of endpoints. Studies by Matsumoto and colleagues have shown that X-irradiation activates iNOS as early as $3 \mathrm{~h}$ post-irradiation and results in an increase in radioresistance among bystander cells (Matsumoto et al 2001). In bystander cells treated with the NO scavenger 2-(4-carboxyphenyl)-4, 4, 5, 5-tetramethylimidazoline-1-oxyl-3-oxide (c-PTIO), the induction of micronuclei (Shao et al 2003, 2004) and $\gamma \mathrm{H} 2 \mathrm{AX}$ foci (Han et al 2007) was significantly reduced, suggesting the role of $\mathrm{NO}$, particularly the constitutive form, in mediating bystander effects.

\section{Role of mitochondria in the bystander response}

The observations that extracellularly applied antioxidant enzymes such as SOD (Yang et al 2005) and catalase (Lyng etal 2006) can inhibit the medium-mediated bystander response suggests a role for reactive radical species in the bystander process. Since mitochondria are the main source of energy production, as well as generators of free radicals in cells, particularly in pathological and stressful conditions, they are the prime target for the source of these radical species. There is recent evidence that point mutations in the mitochondrial genome (Murphy et al 2005), as well as an increase in mitochondrial mass (Nugent et al 2007), are induced in directly irradiated human papillomavirus-immortalized human keratinocytes exposed to a 5 Gy dose of gamma-rays or by exposure to bystander factor(s) obtained from such cells. Studies using human fibroblasts that are devoid of mitochondrial DNA and therefore have reduced mitochondrial DNA function have provided evidence that mitochondria play an important role in the regulation of radiation-induced bystander effects (Zhou et al 2008).

\section{$\rho^{\circ}$ Cells show a higher bystander response than wild-type $\rho^{+}$cells}

To explore the role of mitochondria in the radiation-induced bystander effect, a microbeam was used to lethally irradiate either wild-type $\left(\rho^{+}\right)$or mitochondrial-DNA-depleted $\left(\rho^{0}\right)$ cells with $20 \alpha$-particles each in a mixed confluent culture, 
and the bystander response was determined in the non-irradiated fraction. When compared with $\rho^{+}$cells, $\rho^{0}$ cells showed a higher bystander HPRT mutagenic response in a confluent monolayer when $10 \%$ of the same population was lethally irradiated (Zhou et al 2008). However, using mixed cultures of $\rho^{0}$ and $\rho^{+}$cells and targeting only one population of cells with a lethal dose of $\alpha$-particles, a decreased bystander mutagenesis was uniformly found with both cell types, indicating that mitochondrial-deficient cells cannot effectively communicate the bystander signals to wild-type cells. Alternatively, signals from one cell type may modulate expression of the bystander response in another cell type.

\section{The unifying model of bystander effect}

The mechanism of the radiation-induced bystander effect, whether involving cell-to-cell contact or mediated by soluble factors, is not clear and is likely to be complex, involving multiple pathways. It is clear, however, that $p 53$ gene function is not necessary for the effect, since cells without normal p53 function (such as $\mathrm{CHO}$ cells) show a large bystander response in either bystander pathway. It is likely that multiple signalling cascades involving both an initiating event and downstream signalling steps are necessary to mediate the bystander process. Previous studies have shown that COX-2 is critically linked to the radiation-induced bystander effect in normal human fibroblasts (Zhou et al 2005). There is evidence that $\mathrm{NO}$ can induce expression of $C O X-2$ in mouse skin and cultured human airway epithelial cells, and that the NF- $\kappa \mathrm{B}$ pathway is involved in the process (Watkins et al 1997; Chun et al 2004). The recent finding that Bay 11-7082, a specific IKK/NF- $\kappa$ B inhibitor, can eliminate bystander mutagenesis in both wild-type and $\rho^{0}$ cells highlights the important role of this transcription factor in the bystander phenomenon.

As illustrated in the proposed bystander signalling scheme (Figure 4), the bindings of various cytokines to their respective surface receptors are excellent candidates in mediating bystander effects. Ionizing radiation is a strong inducer of the ataxia telangiectasia mutated (ATM) $-\mathrm{IKK}-\mathrm{NF}-\kappa \mathrm{B}$ signalling pathway (Hacker \& Karin 2006), which is further involved in the up-regulation of $T N F \alpha$ gene expression (Karin 2006). As illustrated, NF- $\kappa \mathrm{B}$ directly controls gene expression of $\mathrm{COX}-2$ and $i N O S$. Secreted or membrane-associated forms of $\mathrm{TNF}_{\alpha}$ could induce bystander effects in non-irradiated cells via activation of $C O X-2$ gene expression, as reported recently (Zhou et al 2008). $C O X-2$ is a member of the COX family of genes,

\section{Mechanism of radiation-induced bystander effects}

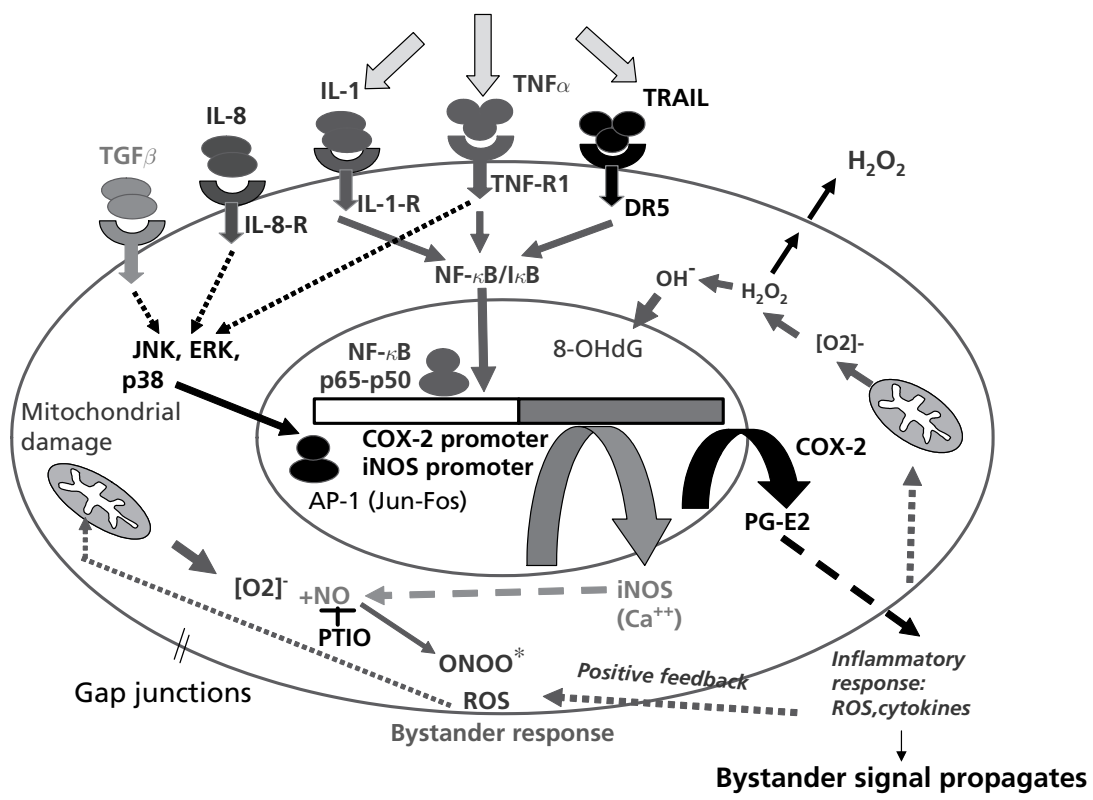

Figure 4 A unifying model of the signalling pathways involved in radiation-induced bystander effects. Expression/secretion of the inflammatory cytokines strongly increased after exposure to ionizing radiation or oxidants. Secreted or membrane-associated forms of cytokines such as tumour necrosis factor (TNF) $\alpha$ activate $\mathrm{I} \kappa \mathrm{B}$ kinase (IKK)-mediated phosphorylation of $\mathrm{I} \kappa \mathrm{B}$, which releases nuclear factor (NF) $-\kappa \mathrm{B}$. NF- $\kappa \mathrm{B}$ enters the nucleus and acts as a transcription factor for cyclooxygense-2 (COX-2) and inducible nitric oxide (NO) synthase (iNOS) genes. TNF $\alpha$ also activates mitogenactivated protein kinase (MAPK) pathways (extracellular signal-related kinase (ERK), c-Jun N-terminal kinase (JNK) and p38) that, via the activation protein (AP)-1 transcription factor, additionally up-regulate expression of COX-2 (Zhou et al 2005) and iNOS, which stimulates production of NO. Mitochondrial damage facilities the production of hydrogen peroxide, which migrates freely across plasma membranes and is subjected to antioxidant removal. Activation of COX-2 provides a continuous supply of reactive radicals and cytokines for the propagation of bystander signals through either gap junctions or medium.

$\mathrm{H}_{2} \mathrm{O}_{2}$, hydrogen peroxide; IL, interleukin; OH; hydroxyl radicals; ONOO; peroxynitrite anions; PTIO, 2-4-(-carboxyphenyl-4,4,5,5,-tetramethylimidazoline-1-oxyl-3-oxide (an NO scavenger); -R, receptor; PG-E2, prostaglandin E2; ROS, reactive oxygen species; TGF, transforming growth factor, TNF, tumour necrosis factor; TRAIL, TNF-related apoptosis-inducing ligand. 
which have important functions in mediating the cellular immune response. Of the two known isoforms, $C O X-2$ is inducible following treatment with different growth factors and cytokines such as transforming growth factor (TGF) $\beta$, TNF $\alpha, \operatorname{IL} 1 \beta$ and different stress factors (Picot \& Garavito 1994). All of these stimuli induce Ras-Raf-MEK-ERK-activation protein (AP)-1 and $\mathrm{IKK}-\mathrm{NF}-\kappa \mathrm{B}$ pathways and finally target COX-2 gene transcription. $\mathrm{COX}-2$ is the initial and rate-limiting enzymatic step in the metabolism of arachidonic acid into a complex group of signalling lipid mediators called prostaglandins (Marnett 1992), which play important roles in modulating cellular inflammation, carcinogenesis and genomic instability. Since the inflammatory response ultimately involves reactive radical species, this provides a positive-feedback mechanism to target mitochondrial and other membranous structures to perpetuate the induction of reactive radical species.

As shown previously in cells with functional mitochondria, up-regulation of $C O X-2$ was detected in both directly irradiated and bystander cells (Zhou et al 2008). Thus, the observation that Bay 11-7082, an inhibitor of IKK-NF- $\kappa$ B activation, can inhibit the bystander response provides clear evidence that the $\mathrm{NF}-\kappa \mathrm{B}-\mathrm{COX}-2-\mathrm{PGE}_{2}$ and $\mathrm{NF}-\kappa \mathrm{B}-\mathrm{iNOS}-\mathrm{NO}$ pathways are critical for the radiation-induced bystander effect in cells with functional mitochondria. However, in $\rho^{0}$ cells, the contribution of COX-2 to the bystander process is less pronounced, while the NF- $\kappa \mathrm{B}-\mathrm{iNOS}-\mathrm{NO}$ pathway actively operates, although at lower level compared with normal cells.

Elevated expression of $i N O S$ and $C O X-2$ induces production of NO and reactive oxygen species, which destroy normal mitochondrial function. It is likely that mitochondrial damage results in leakage of mitochondrial membranes, allowing release of superoxide anions into the cytosol and increasing oxidative stress on the cells (Agarwall \& Sohal 1994; Wei 1998). The observation that SOD and catalase, both large molecules that do not enter the cells freely, are able to reduce the bystander responses is consistent with the following sequence of events in the generation of oxyradicals: superoxide $\rightarrow$ hydrogen peroxide $\rightarrow$ hydroxyl radicals. Hydrogen peroxide can move freely between intracellular and extracellular compartments, and its removal in the presence of extracellularly applied catalase and SOD results in a decrease in intracellular oxidative stress. This decrease in oxidant level may reduce mitochondrial membrane damage and a corresponding decrease in the bystander response.

\section{Conclusion}

The radiation-induced bystander effect is strictly a low-dose phenomenon since at high doses the bystander effect observed at low doses will be largely overshadowed by direct damage to cells. The bystander effect contributes to the debate as to the validity of the linear no-threshold model for low-dose radiation risk assessment by implying that the biological effects of low doses, where not all cells are traversed by a charged particle, are amplified by the transfer of factors to non-irradiated neighbours. Previous findings that mutations in the nuclei of hit cells can be induced by targeted cytoplasmic irradiation (Wu et al 1999), which can further result in a bystander effect in mammalian cells (Shao et al 2006), suggest that the radiation-sensitive target is more than just the nucleus. The abscopal or out-of-field phenomenon would imply that bystander effects coordinate a complex interplay involving organs, tissues and cells. Although many of the bystander responses reported thus far have been detrimental in nature (e.g. oncogenic transformation, mutations and chromosomal aberrations) (see Hall \& Hei 2003, Morgan 2003 for reviews), protective effects have also been reported (e.g induction of terminal differentiation) (Belyakov et al 2006) and apoptosis of potentially damaged cells (Coates et al 2004). On the other hand, there is evidence that bystander cells also show increased genomic instability, a predisposing factor for carcinogenesis. Hence the contribution of bystander effects in radiation risk assessment has to be evaluated in terms of tissue context, the phenotypic behaviour of their progeny and the presence of other competing low-dose effects, which include adaptive response, genomic instability and individual genetic susceptibility.

Thus far, most of the published data on bystander effects have been largely phenomenological in nature. In the future, mechanistic-based studies that can provide insight into the nature of the signalling molecule(s), the clinical relevance of the bystander effects and ways in which the bystander phenomenon can be manipulated to increase therapeutic gain in radiotherapy should be considered as priorities for investigators in the field.

\section{References}

Agarwal, S., Sohal, R. S. (1994) DNA oxidative damage and life expectancy in houseflies. Proc. Natl Acad. Sci. USA 91: 12332-12335

Azzam, E. I., de Toledo, S. M., Gooding, T., Little, J. B. (1998) Intercellular communication is involved in the bystander regulation of gene expression in human cells exposed to very low fluences of alpha particles. Radiat. Res. 150: 497-504

Azzam, E. I., de Toledo, S. M., Waker, A. J., Little, J. B. (2000) High and low fluences of alpha-particles induce a G1 checkpoint in human diploid fibroblasts. Cancer Res. 60: 2623-2631

Azzam, E. I., De Toledo, S. M., Spitz, D. R., Little, J. B. (2002) Oxidative metabolism modulates signal transduction and micronucleus formation in bystander cells from alpha-particle-irradiated normal human fibroblast cultures. Cancer Res. 62: 5436-5442

Belyakov, O. V., Folkard, M., Mothersill, M., Prise, K. M., Michael, B. D. (2006) Bystander induced differentiation: a major response to targeted irradiation of a urothelial explant model. Mutat. Res. 597: 43-49

Bruzzone, R., Meda, P. (1988) The gap junction: a channel for multiple functions? Eur. J. Clin. Invest. 18: 444-453

Chun, K-S., Cha, H.-H., Shin, J.-W., Na, H.-K., Park, K.-K., Chung, W.-Y., Surh, Y.-J. (2004) Nitric oxide induces expression of cyclooxygenase-2 in mouse skin through activation of NF- $\kappa \mathrm{B}$. Carcinogenesis 25: 445-454

Coates, P. J., Lorimore, S. A., Wright, E. G. (2004) Damaging and protective cell signaling in the untargeted effects of ionizing radiation. Mutat. Res. 568: 5-20

Dale, W. M. (1940) The effects of X-rays on enzyme. Biochem. J. 34: 1367-1373

Dowling-Warriner, C. V., Trosko, J. E. (2000) Induction of gap junctional intercellular communication, connexin 43 expression, and subsequent differentiation in human fetal neuronal cells by stimulation of the cyclic AMP pathway. Neuroscience 95: 859-868 
El-Fouly, M. H., Trosko, J. E., Chang, C. C. (1987) Scrapeloading and dye transfer. A rapid and simple technique to study gap junctional intercellular communication. Exp. Cell Res. 168: $422-430$

Geard, C. R., Jenkins-Baker, G., Marino, S., Ponnaiya, B. (2002) Novel approaches with track segment alpha particles and cell co-cultures in studies of bystander effects. Radiat. Protect. Dosimet. 99: 233-236

Hacker, H., Karin, M. (2006) Regulation and function of IKK and IKK-related kinases. Sci. STKE 357: re13

Hall, E. J., Hei, T. K. (2003) Genomic instability and bystander effects induced by high LET radiation. Oncogene 22: 7034-7042

Han, W., Wu, L., Chen, S., Bao, L., Zhang, L., Jiang, E., Zhao, Y., $\mathrm{Xu}$, A., Hei, T. K., Yu, Z. (2007) Constitutive nitric oxide acting as a possible intercellular signaling molecule in the initiation of radiation-induced DNA double strand breaks in non-irradiated bystander cells. Oncogene 26: 2330-2339

Hu, B., Han, W., Wu, L., Feng, H., Liu, X., Zhang, L., Xu, A., Hei, T. K., Yu, Z. (2005) In situ visualization of DSBs to assess the extranuclear/extracellular effects induced by low-dose alpha-particle irradiation. Radiat. Res. 164: 286-291

Hu, B., Wu, L., Han, W., Zhang, L., Chen, S., Xu, A., Hei, T. K., Yu, Z. (2006) The time and spatial effects of bystander response in mammalian cells induced by low dose radiation. Carcinogenesis 27: 245-251

International Commission on Radiological Protection (2005) Low dose extrapolation of radiation related cancer risk. Pergamon, New York, Report no. 99

Karin, M. (2006) Nuclear factor-kappaB in cancer development and progression. Nature 441: 431-436

Kotval, J. P., Gray, L. H. (1947) Structural changes produced in microspores of Tradescantia by $\alpha$-particles. J. Genet. 48: 135-154

Koyama, S., Kodama, K., Suzuki, K., Matsumoto, T., Miyazaki, T., Watanabe, M. (1998) Radiation induced long lived radicals which cause mutation and transformation. Mutat. Res. 421: 45-54

Lea, D. E., Smith, K. M., Holmes, B., Markham R. (1944) Direct and indirect actions of radiation on viruses and enzymes. Parasitology 36: $110-118$

Lewis, D. A., Mayhugh, B. M., Qin, Y., Trott, K., Mendoca, M. S. (2001) Production of delayed death and neoplastic transformation in CGL1 cells by radiation-induced bystander effects. Radiat. Res. 156: $251-258$

Lyng, F. M., Seymour, C. B., Mothersill, C. (2000) Production of a signal by irradiated cells which leads to a response in unirradiated cells characteristic of initiation of apoptosis. Br. J. Cancer $\mathbf{8 3}$ : 1223-1230

Lyng, F. M., Maguire, P., McClean, B., Seymour, C., Mothersill, C. (2006) The involvement of calcium and MAP kinase signaling pathways in the production of radiation-induced bystander effects. Radiat. Res. 165: 400-409

Marnett, L. J. (1992) Aspirin and the potential role of prostaglandins in colon cancer. Cancer Res. 52: 5575-5589

Matsumoto, H., Hayashi, S., Hatashita, M., Ohnishi, K., Shioura, H., Ohtsubo, T., Kitai, R., Ohnishi, T., Kano, E. (2001) Induction of radioresistance by a nitric oxide-mediated bystander effect. Radiat. Res. 155: 387-396

Morgan, W. F. (2003) Non-targeted and delayed effects of exposure to ionizing radiation: I. Radiation induced genomic instability and bystander effects in vitro. Radiat. Res. 159: 567-580

Mothersill, C., Seymour, C. (1997) Delayed expression of lethal mutations and genomic instability in the progeny of human epithelial cells that survived in a bystander-killing environment. Radiat. Oncol. Investig. 5: 106-110

Murphy, J. E., Nugent., S., Seymour, C., Mothersill, C. (2005) Mitochondrial DNA point mutations and a novel deletion induced by direct low-LET radiation and by medium from irradiated cells. Mutat. Res. 585: 127-136
Nagar, S., Smith, L. E., Morgan, W. F. (2003) Characterization of a novel epigenetic effect of ionizing radiation: the death inducing effect. Cancer Res. 63: 324-328

Nagasawa, H., Little, J. (1992) Induction of sister chromatid exchanges by extremely low doses of alpha-particles. Cancer Res. 52: 6394-6396

Narayanan, P. K., Goodwin, E. H., Lehnert, B. E. (1997) Alpha particles initiate biological production of superoxide anions and hydrogen peroxide in human cells. Cancer Res. 57: 3963-3971

Nugent, S. M., Mothersill, C. E., Seymour, C., McClean, B., Lyng, F. M., Murphy, J. E. (2007) Increased mitochondrial mass in cells with functionally compromised mitochondria after exposure to both direct gamma radiation and bystander factors. Radiat. Res. 168: 134-142

Picot, D., Garavito, R. M. (1994) Prostaglandin H synthase: implications for membrane structure. FEBS Lett. 346: 21-25

Preston, R. J. (2005) Bystander effects, genomic instability, adaptive response, and cancer risk assessment for radiation and chemical exposures. Toxicol. Appl. Pharmacol. 207(2 Suppl): 550-556

Shao, C., Stewart, V., Folkard, M., Michael, B. D., Prise, K. M. (2003) Nitric oxide-mediated signaling in the bystander response of individually targeted glioma cells. Cancer Res. 63: 8437-8442

Shao, C., Folkard, M., Michael, B. D., Prise, K. M. (2004) Targeted cytoplasmic irradiation induces bystander responses. Proc. Natl Acad. Sci. USA 101: 13495-13500

Shao, C., Lyng, F. M., Folkard, M., Prise, K. M. (2006) Calcium fluxes modulate the radiation-induced bystander responses in targeted glioma and fibroblast cells. Radiat. Res. 166: 479-487

Suzuki, M., Zhou, H., Geard, C. R., Hei, T. K. (2004) Effect of medium on chromatin damage in bystander mammalian cells. Radiat. Res. 162: 264-269

Trosko, J. E. (1998) Hierarchical and cybernetic nature of biologic systems and their relevance to homeostatic adaptation to low-level exposures to oxidative stress-inducing agents. Environ. Health Perspect. 106 (Suppl 1): 331-339

Watkins, D. N., Garlepp, M., Thompson, P. J. (1997) Regulation of the inducible cyclo-oxygenase pathway in human cultured airway epithelial (A549) cells by nitric oxide. Br. J. Pharmacol. 121: $1482-1488$

Wei, Y. H. (1998) Oxidative stress and mitochondrial DNA mutations in human aging. Proc. Soc. Exp. Biol. Med. 217: 53-63

Wu, L. J., Randers-Pehrson, R., Xu, A., Waldren, C. A., Geard, C. R., Yu, Z. Y., Hei, T. K. (1999) Targeted cytoplasmic irradiation with alpha particles induces mutations in mammalian cells. Proc. Natl Acad. Sci. USA 96: 4959-4964

Yang, H., Asaad, N., Held, K. D. (2005) Medium-mediated intercellular communication is involved in bystander responses of X-rayirradiated normal human fibroblasts. Oncogene 24: 2096-2103

Zhou, H., Randers-Pehrson, G., Waldren, C. A., Vannais, D., Hall, E. J., Hei, T. K. (2000) Induction of a bystander mutagenic effect of alpha particles in mammalian cells. Proc. Natl Acad. Sci. USA 97: 2099-2104

Zhou, H., Suzuki, M., Randers-Pehrson, G., Vannais, D., Waldren, C. A., Cheng, G., Trosko, J. E., Hei, T. K. (2001) Radiation risk to low fluences of alpha particles may be greater than we thought. Proc. Natl Acad. Sci. USA 98: 14410-14415

Zhou, H., Suzuki, M., Geard, C. R., Hei, T. K. (2002) Effects of irradiated medium with or without cells on bystander cell responses. Mutat. Res. 499: 135-141

Zhou, H., Ivanov, V. N., Gillespie, J., Geard, C. R., Amundson, S. A., Brenner, D. J., Yu, Z., Lieberman, H. B., Hei, T. K. (2005) Mechanism of radiation-induced bystander effect: role of the cyclooxygenase-2 signaling pathway. Proc. Natl Acad. Sci. USA 102: $14641-14646$

Zhou, H., Ivanov, V. N., Lien, Y. C., Davidson, M., Hei, T. K. (2008) Mitochondrial function and NF- $\kappa$ B mediated signaling in radiation-induced bystander effects. Cancer Res. 68: 2233-2240 